\title{
Synovium, synergy and serendipity
}

\author{
Gregory M. Pastores
}

Published online: 27 May 2011

(C) SSIEM and Springer 2011

Certain forms of arthritis, such as juvenile idiopathic arthritis (JIA) and osteoarthritis (OA), are believed to be influenced by genetic factors. These conditions are often associated with significant health and productivity costs, in addition to the outlay incurred as a result of medical management of the disease. Thus, substantial resources have been put into identifying causal genetic defects and/or susceptibility alleles for JIA and OA, with the majority of recent endeavors employing genome-wide association scan (GWAS) technology or a candidate gene discovery approach. It is hoped these strategies would provide insight into pathology, which would in turn rationalize and focus therapeutic approaches.

Recent reviews on the genetics of arthritis have pointed out that associations have been observed with JIA and variants at $H L A, P T P N 22$ and several other genes; (Angeles-Han and Prahalad 2010) whereas alleles at three loci (i.e., the 7q22 locus, GDF5 and DIO2) have been associated with OA (Loughlin 2011). Although GWAS has successfully revealed numerous susceptibility genes for certain forms of arthritis, odds ratios associated with risk alleles are generally low and account for only a small proportion of estimated heritability. Furthermore, it should be pointed out that although GWAS may help to identify chromosomal regions of interest, definitive identification of causal gene defects and attendant pathomechanisms require further lines of investigation.

As with other disorders with overlapping phenotypes and broad heterogeneity in causal gene defects, it is likely a subset

G. M. Pastores $(\square)$

Departments of Neurology and Pediatrics,

New York University School of Medicine,

New York, USA

e-mail: Gregory.Pastores@nyumc.org of patients with familial clustering of JIA or OA may be due to rare alleles with a large effect (Tsuji 2010). In the current issue of the Journal, Imundo and colleagues (DOI 9343) describe a consanguineous family with three affected children (ages 11, 13 and 22) whose chronic large joint disease has been ascribed to a HYAL1 (hyaluronidase-1) gene defect (Imundo et al. 1995). The familial occurrence and combination of findings, such as the widespread proliferative synovitis (evident on both MRI and ultrasound studies, and which on biopsy revealed membrane-bound vacuoles in histiocytes) prompted a search for a diagnosis other than JIA. Causality was discovered by means of homozygosity mapping, illustrating the power of a genotype-driven approach using currently available tools to establish an etiologic diagnosis even within a single family. In a prior era, characterization of the storage material would have been performed, followed by a search for the enzyme or transport protein whose dysfunction would be implicated. By sequence analysis, the affected children were shown to be homozygous for a HYAL1 deletion (c.104delT) mutation, which corresponded with an absence of hyaluronoglucosaminidase activity. These findings establish this family as the second case of Mucopolysaccharidosis type IX (MPS-IX, Natowicz syndrome) (Imundo et al. 1995). As was the case with Natowicz's patient, analyses of urinary mucopolysaccharides (glycosaminoglycans) and oligosaccharides were normal. Moreover, none of these patients exhibited clinical signs of neurological or visceral involvement that would have pointed to a lysosomal storage disorder.

The first reported patient was a 14-year-old girl who presented with multiple periarticular masses, mild short stature and dysmorphic features (e.g., flat nasal bridge, bifid uvula, submucosal cleft palate) (Natowicz et al. 1996). In contrast, the patients in the current report appeared to have isolated joint disease, were of normal stature and had no 
signs of cutaneous swelling or otolaryngeal problems (Imundo et al. 1995). The relatively more severe presentation in the first case report may partly be accounted for by the combination of mutations indentified (i.e., a missense $[1412 \mathrm{G} \rightarrow \mathrm{A}]$ mutation and complex insertion/deletion [1361del37ins14] causing a premature termination in HYAL1 gene) (Triggs-Raine B et al. 1999).

Hyaluronidase-1 (HYAL1) is one of several lysosomal endogylcosidases required for the breakdown of hyaluronan, a high molecular mass glycosaminoglycan that is abundant in the extracellular matrix of connective tissues. The encoding gene (HYAL1) has been mapped to human chromosome 3p21.3 (Csóka et al. 1998). In 2008, Martin and colleagues reported the phenotype of a mouse model completely deficient in Hyall activity (Martin et al. 2008). The affected mouse showed agedependent bony outgrowths and premature loss of the articular cartilage proteoglycans, but no joint masses or serum elevations in hyaluronan. The limited number of human subjects does not enable genotype-phenotype correlations, although further biochemical studies may provide insight into the downstream and related events leading to disease expression.

There is a second lysosomal hyaluronidase (HYAL2), which also maps to 3p21.3, but with different substrate specificities (Lepperdinger et al. 1998). HYAL2-deficient mice generated through a conditional Cre-lox system exhibited craniovertebral bone defects, but no hyaluronan accumulation in tissues, including bones; however, plasma hyaluronan levels were increased (Jadin et al. 2008). To date, no human patients have been identified with HYAL2 gene defects. So, the challenge is set for clinicians to identify the first case. Meanwhile, several lessons can be drawn from the experience reported by Imundo and colleagues: 1) how thoughtful clinical characterization of patients can separate the rare from the common, 2) the power of a genotype-driven approach in the discovery of cause, and 3) the potential higher yield of pursuing common disease by searching for rare variants versus seeking for associations. With technical advances in nextgeneration sequencing methods, allowing for decreased costs clinical practice in rare disease medicine may likely involve personalized genome testing.

\section{References}

Angeles-Han S, Prahalad S (2010) The genetics of juvenile idiopathic arthritis: what is new in 2010? Curr Rheumatol Rep 12(2):87-93

Loughlin J (2011) Osteoarthritis year 2010 in review: genetics. Osteoarthritis Cartilage 19(4):342-5

Tsuji S (2010) Genetics of neurodegenerative diseases: insights from high-throughput resequencing. Hum Mol Genet 19(R1):R65-70

Imundo et al. (1995) Proc Natl Acad Sci USA 92:3019-23

Natowicz MR, Short MP, Wang Y, Dickersin GR, Gebhardt MC, Rosenthal DI, Sims KB, Rosenberg AE (1996) Clinical and biochemical manifestations of hyaluronidase deficiency. N Engl J Med 335(14):1029-33

Triggs-Raine B, Salo TJ, Zhang H, Wicklow BA, Natowicz MR (1999) Mutations in HYAL1, a member of a tandemly distributed multigene family encoding disparate hyaluronidase activities, cause a newly described lysosomal disorder, mucopolysaccharidosis IX. Proc Natl Acad Sci USA. 96(11):6296-300.

Csóka AB, Frost GI, Heng HH, Scherer SW, Mohapatra G, Stern R (1998) The hyaluronidase gene HYAL1 maps to chromosome 3p21.2-p21.3 in human and 9 F1-F2 in mouse, a conserved candidate tumor suppressor locus. Genomics 48(1):63-70

Martin DC, Atmuri V, Hemming RJ, Farley J, Mort JS, Byers S, Hombach-Klonisch S, Csoka AB, Stern R, Triggs-Raine BL (2008) A mouse model of human mucopolysaccharidosis IX exhibits osteoarthritis. Hum Mol Genet 17(13):1904-15

Lepperdinger G, Strobl B, Kreil G (1998) HYAL2, a human gene expressed in many cells, encodes a lysosomal hyaluronidase with a novel type of specificity. J Biol Chem 273(35):22466-70

Jadin L, Wu X, Ding H, Frost GI, Onclinx C, Triggs-Raine B, Flamion B (2008) Skeletal and hematological anomalies in HYAL2-deficient mice: a second type of mucopolysaccharidosis IX? FASEB J 22(12):4316-26 